\title{
Revisión de Formación Ética en La Universidad Nacional de Colombia, Sede Medellín
}

\author{
Review Of Ethical Training At The Universidad Nacional De Colombia, \\ Campus Medellin
}

\author{
Susana Valencia Rodríguez* \\ Miguel Rojas López** \\ Estefanía Vera Aguirre**
}

\begin{abstract}
Resumen: La formación ética en la Universidad Nacional de Colombia, Sede Medellín -UN-, no se evidencia dentro de los planes curriculares como una asignatura obligatoria ni disciplinar. Para el año 2017 sólo se registran en el sistema de información académico- SIA- 2 materias fundamentadas en esta temática. Para esta investigación inicialmente se realiza una identificación de las técnicas metodológicas empleadas por las entidades de educación superior en la ciudad de Medellín, Colombia. Posteriormente se procede a realizar un análisis cualitativo de las asignaturas dictadas que poseen relación con las ciencias éticas y que son ofrecidas en los planes de estudio de los programas de pregrado. Se expone la metodología sugerida a seguir para la enseñanza de materias con enfoque social y finalmente se diseña una asignatura ética que presente metodología adaptativa para ser impartida en los programas de pregrado, inicialmente Ingeniería Administrativa e Ingeniería Industrial de la UN.
\end{abstract}

Palabras clave: Ética, Formación, Metodología Académica, Asignatura, Universidad Nacional de Colombia.

Abstract: The ethical education at the Universidad Nacional de Colombia, Campus Medellín - UN-, not evidenced within the curricular plans as a compulsory subject or discipline. For the year 2017 only register in the academic information system -SIA-, 2 subjects based on this thematic. For this research is initially an identification of the methodological techniques employed by institutions of higher education in the city of Medellin, Colombia. Subsequently is to perform a qualitative analysis of the taught subjects that have relationship with the ethical science and which are offered in the curricula of undergraduate programs. The suggested methodology is exposed to the teaching of subjects with a social focus and finally an ethical subject which present an adaptive methodology to be taught in the undergraduate programs, initially Administrative Engineering and Industrial Engineering at the UN.

Keywords: Ethics, Training, Academic methodology, Subject, Universidad Nacional de Colombia.

JEL: Z13

*Ingeniera Administradora, Universidad Nacional de Colombia, sumvalenciaro@unal.edu.co. Enlace ORCID: https://orcid.org/0000-0002-6113-0939

**Profesor Asociado, Universidad Nacional de Colombia, mdrojas@unal.edu.co. Enlace ORCID: https://orcid.org/0000-0002-3531-4910

*** Ingeniera Administradora, Universidad Nacional de Colombia, everaa@unal.edu Enlace ORCID: https://orcid.org/0000-0002-6113-0939 


\section{Examen de la formation en éthique à l'Université nationale de Colombie, siège de Medellin}

Résumé: La formation en éthique à l'Université nationale de Colombie, Campus de Medellin UN-, n'est pas évidente dans les plans d'études en tant que matière obligatoire ou disciplinaire. Pour l'année 2017, seuls les sujets SIA-2 basés sur ce sujet sont enregistrés dans le système d'information académique. Pour cette recherche, une identification des techniques méthodologiques utilisées par les entités d'enseignement supérieur dans la ville de Medellin, en Colombie, est initialement réalisée. Par la suite, une analyse qualitative des matières enseignées qui sont liées aux sciences éthiques et qui sont offertes dans les curricula des programmes de premier cycle est réalisée. La méthodologie suggérée à suivre pour l'enseignement de sujets à orientation sociale est exposée et enfin un sujet éthique est conçu qui présente une méthodologie adaptative à enseigner dans les programmes de premier cycle, initialement l'ingénierie administrative et le génie industriel de l'UN.

Mots-clés: Éthique, formation, méthodologie académique, sujet, Université nationale de Colombie.

\section{Revisão de Treinamento Ético na Universidade Nacional da Colômbia, Sede em Medellin}

Resumo: O treinamento de ética na Universidade Nacional da Colômbia, Campus Medellin-UN, não é evidente nos planos curriculares como disciplina obrigatória ou disciplinar. Para o ano de 2017, somente os sujeitos do SIA-2 com base nesse assunto estão cadastrados no sistema de informações acadêmicas. Para esta pesquisa, uma identificação das técnicas metodológicas utilizadas pelas entidades de ensino superior na cidade de Medellín, Colômbia, é inicialmente realizada. Posteriormente, é realizada uma análise qualitativa das disciplinas ensinadas que são relacionadas às ciências éticas e que são oferecidas nos currículos dos cursos de graduação. Sugere-se a metodologia sugerida a ser seguida para o ensino de disciplinas com enfoque social e, por fim, é desenhado um tema ético que apresenta metodologia adaptativa para ser ministrado nos cursos de graduação, inicialmente em Engenharia Administrativa e Engenharia Industrial da UN.

Palavras-chave: Ética, Formação, Metodologia Acadêmica, Assunto, Universidade Nacional da Colômbia. 


\section{Introducción}

Se hace referencia a las metodologías utilizadas por algunas instituciones de educación superior en la ciudad de Medellín con fines académicos en asignaturas de formación ética. Entre las características evaluadas se destacan: educación virtual y presencial, cátedras, intensidad horaria, cantidad de asignaturas ofertadas en el programa académico, certificación de la institución educativa, entre otros.

La investigación surge del análisis a situaciones problemáticas presentadas en el siglo XXI, donde las decisiones de juicio ético pueden ser las causantes de problemáticas sociales y conflictos de intereses.

El artículo se enfoca en estudiantes universitarios como base social, moral, tecnológica y cultural de la ciudad, buscando para ellos un desarrollo profesional adecuado que integre todas las áreas de crecimiento personal comunicación, creatividad, técnica, humanidad, sociabilidad y superación personal.

Se plantea una propuesta de asignatura para estudiantes de áreas administrativas, ingenieriles y productivas, buscando el desarrollo de competencias humanas y formación del carácter basado en metodologías de enseñanza cooperativa con foco en discusión y análisis grupal.

\section{Enseñanza - Aprendizaje En La Ciudad de Medellín}

Se define el modelo metodológico de enseñanza y aprendizaje empleado en las Universidades de la ciudad de Medellín; basado en recopilación de estudios teóricos y prácticos realizados por algunos autores.

La definición de educación es:

"Los conocimientos que se diseñan a la medida, direccionada al desarrollo de las habilidades únicas de cada estudiante y a proporcionar una experiencia emocional positiva. Una enseñanza que evalúa el talento natural y la forma en que un estudiante aprende" (Serna M, 2015). 


\section{Contexto valencia, S. Rojas, M. . y vera, E.}

Se establecen los métodos pedagógicos del sistema educativo colombiano, con foco en las universidades de Medellín; los cuales, históricamente se han visto afectados por la era industrial y la revolución tecnológica, épocas donde el mercado establecía necesidades basado en la capacidad productiva de las empresas, impulsando a educar trabajadores competentes, "obreros" listos para responder a la necesidad laboral del momento, que permitiera una cantidad de títulos con el menor esfuerzo. Las características de este sistema son (Serna M, 2015):

- La responsabilidad de la educación se distribuye entre padres, gobierno, sociedad e individuos.

- Los temas comunes de enseñanza son: destrezas prácticas, conocimiento interdisciplinar y capacidad de aprender.

- La metodología principal es memorística.

- La verificación de lo aprendido se da por observación y por evaluaciones integrales.

- La ubicación del aprendizaje son las escuelas acreditadas y diseñadas para educar - universidades, instituciones universitarias, centros de aprendizaje, entre otros-.

- Se tiene la posibilidad de aprender de diferentes personas -varios puntos de vista, diversos profesores- y tomar ideales de todos ellos para definir la opinión propia.

Este tipo de sistema educativo se centra en el aprendizaje memorístico, pretende retener información exacta en el cerebro, usando la repetición como mecanismo. Puede ser, pasivo cuando la comprensión de la información que se da es baja o nula y solo es capaz de mencionar pocos hechos o detalles de importancia de lo enseñado; y activo cuando el nivel de comprensión es superficial y es capaz de proporcional descripciones básicas y adecuadas de los temas enseñados (Buendía Eisman \& Olmedo Moreno, 2002).

Dicho sistema educativo, desarrolla en los estudiantes un proceso de aprendizaje académico afectado por tres dimensiones, la conciencia que hay en el acto de aprendizaje, el contenido de la materia o la información que se 
Revisión de Formación Ética en la Universidad...

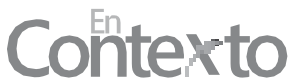

está enseñando y el contexto que rodea el acto de aprendizaje (Marton \& Lennart, 1979):

1. Conciencia del acto de aprendizaje: característica interna del estudiante, es subjetiva y diferente para cada persona, se refiere a la predisposición del estudiante ante la asignatura.

2. Contenido de la materia: es una cualidad externa, puede ser influenciada por el profesor o encargado del curso, incluye los temas que se tratarán en la asignatura.

3. Contexto de aprendizaje: tiene rasgos internos y externos, es decir, rasgos en los cuales el sistema puede intervenir y otros innatos en las cualidades del estudiante. Hace referencia al sistema de evaluación del curso, las experiencias que se han tenido con el aprendizaje previamente, el tipo de material usado para el aprendizaje y la metodología de enseñanza.

Además, existen tres enfoques con los que se puede abordar el aprendizaje de una tarea(Buendía Eisman \& Olmedo Moreno, 2002) (Entwistle, 1991):

- Aprendizaje superficial: se desarrolla para cubrir con las necesidades institucionales.

- Aprendizaje profundo: aprendizaje para el propio desarrollo personal.

- Aprendizaje estratégico: es la organización del tiempo y el espacio de trabajo, la consecución del programa de estudios de manera eficaz.

El enfoque de aprendizaje mayoritariamente usado por los estudiantes universitarios, según un estudio descriptivo realizado en la universidad de granada en el año 2002 es el Enfoque de Aprendizaje Superficial; ya que la motivación para aprender es aprobar el curso y el miedo constante al fracaso educativo, donde la intención es cubrir los requisitos mínimos de las tareas asignadas por medio de procesos memorísticos (Buendía Eisman \& Olmedo Moreno, 2002).

Lo anterior, señala los errores del sistema educativo y cuestiona la eficiencia de los mecanismos pedagógicos actuales para la formación de técnicos, tecnólogos y profesionales.

Sin embargo, los resultados se ven modificados dependiendo del pregrado que se está cursando; los estudiantes desarrollan un método de aprendizaje más 
Valencia, S., Rojas, M. y Vera, E.

efectivo (usando el enfoque estratégico) donde su motivación para estudiar está en obtener buenas calificaciones y competir con otros

estudiantes, esto combinando los procesos de aprendizaje operativo, comprensivo y memorístico (Buendía Eisman \& Olmedo Moreno, 2002).

Además, de los problemas mencionados sobre la metodología del sistema educativo, existen otros posibles inconvenientes generados por el subdesarrollo del país. A continuación se enumeran algunos aspectos relevantes de la situación (Molina Álvarez, 1999):

1. Las universidades, públicas y privadas de países sub-desarrollados tienen enfoques pedagógicos atrasados y deficientes, orientados a funciones concretas y operativas.

2. La mayor parte de las instituciones carecen de la preparación profesional adecuada, limitando la educación al "sujeto que enseña", dejando a un lado el "sujeto que aprende".

3. Los egresados son dependientes, poco creativos y con problemas para tomar decisiones.

4. La enseñanza es tradicional y el profesor es el centro del proceso de aprendizaje.

5. Los planes de estudio y programas son fragmentados, no poseen un hilo conductor ni una lógica adecuada que facilite la integración de información.

6. El enfoque principal está en 'aprender a hacer' y 'aprender a conocer', dejando de un lado el 'aprender a convivir' y 'aprender a ser'.

7. No hay una estrategia de aprendizaje para los estudiantes, así que ellos desarrollan estrategias intuitivas, haciendo más ineficiente el proceso.

8. Los docentes en ocasiones carecen de conocimientos pedagógicos y metodológicos.

9. En muchas instituciones no existe la evaluación institucional enfocada a la mejora continua de sus programas.

10. No está definido correctamente el perfil profesional que se desea obtener.

\section{Enseñanza - Aprendizaje de Ciencias Éticas}

Luego de identificar el modelo educativo de las universidades de Medellín, se procede con el desarrollo de una asignatura -lineamientos básicos, temas a 
Revisión de Formación Ética en la Universidad... tratar, metodología, material de estudio- que pretende cumplir con todos los aspectos de la definición de Educación, mencionada al inicio del texto.

Para iniciar, se observa en la Figura. 1, las competencias profesionales de un egresado universitario, reflejadas en compromiso social, conocimientos técnicos, auto-superación permanente, iniciativa y creatividad para desarrollar tareas, competencias comunicativas y valores humanos que posee.

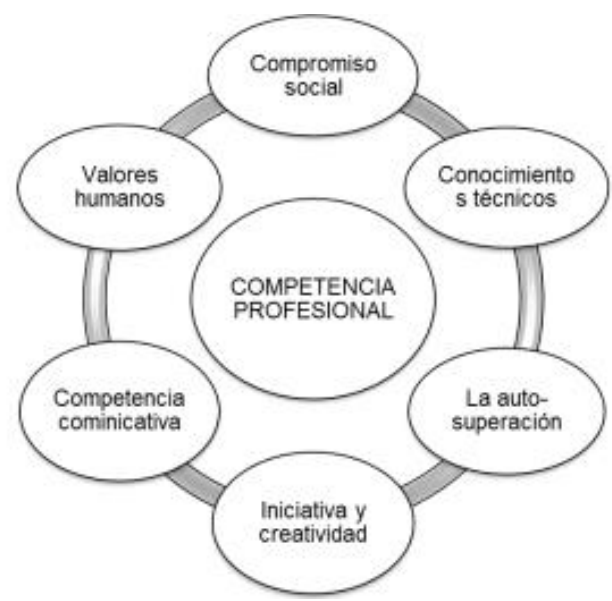

Figura 1. Componentes de la competencia profesional.

Molina Álvarez, 1999

Para el correcto desarrollo de estas competencias, es apropiado generar nuevas metodologías de enseñanza, que favorezcan el aprendizaje, que se acoplen a la globalización y al cambio continuo en los diferentes aspectos de vida, permitiendo la formación de profesionales, con capacidad de análisis, críticos, auto-didactas e integrales.

Para lograrlo, se debe enfatizar el ámbito técnico y social de los estudiantes; es necesario formar seres humanos íntegros, con énfasis en el convivir y el ser. Profesionales que no dejen de lado los valores humanos y el compromiso social para forjar su competencia profesional.

Por esta razón, las universidades no pueden limitarse a impartir sólo conocimientos técnicos y teóricos; deben formar futuros ciudadanos que promuevan los derechos humanos y el bien común (Serna M, 2015)(Zerpa, 2012). 


\section{Contexto valencia, S., Rojas, M. .y Vera, E}

Esto cobra fundamental importancia en los planes de formación de profesionales de distintas especialidades que se dictan en las instituciones de educación superior y especialmente en las de corte tecnocientífico. Tomando en consideración que es el avance en la tecnología uno de los más importantes motores de cambio social que ha operado en la historia del mundo y que más ha impactado en las vidas de las personas. Esto justifica la conveniencia de encontrar a la ética en una intersección con los dos campos, del capital social y el desarrollo" (Zerpa, 2012).

Los estudiantes universitarios deben entrenar para tomar "decisiones de juicio ético" en su vida profesional, que lleven a un bien común por encima del personal, dado que "la moral y la ética se articulan con costumbres o prácticas que son comúnmente realizadas por las personas” (Zerpa, 2012) y no siguiendo métodos de aprendizaje memorísticos y repetitivos.

Dicho esto, se plantean las bases para una asignatura de ciencias éticas para el Departamento de Ingeniería de la Organización de la Facultad de Minas en la Universidad Nacional de Colombia - UNAL-, sede Medellín, la cual está compuesta por las carreras Ingeniería Administrativa e Ingeniería industrial.

Para esto, se hace una investigación preliminar que proporcione un panorama general sobre cómo se da el estudio de las ciencias éticas en las universidades de la ciudad.

Ésta investigación se desarrolla en las cinco mejores Universidades de Medellín según el Ranking QS University Rankings: Latin America, el cual califica las universidades según la reputación académica, la reputación de empleados, el tiempo que están los estudiantes y empleados en la institución, el número de citaciones por publicación (papers) del material de la universidad, el porcentaje de empleados con títulos doctorados y finalmente el impacto que tiene la universidad en la web ("QS Top University, 'QS University Rankings: Latin America,”” 2015). Luego de estos resultados, se 
Revisión de Formación Ética en la Universidad...

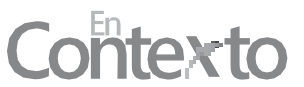

procede con la elaboración del modelo para la asignatura en la Universidad Nacional de Colombia, sede Medellín.

A partir del resultado obtenido en las universidades, se plantean las características de las asignaturas de ciencias éticas que son dictadas actualmente en la ciudad para pregrados administrativos e industriales.

Las cualidades que se evalúan en cada universidad son: la intensidad horaria de la materia, el método de enseñanza y la modalidad en la que es ofrecida. Las universidades valoradas son:

1. Universidad Nacional de Colombia, sede Medellín.

2. Universidad de Antioquia.

3. EAFIT.

4. Universidad Pontificia Bolivariana.

5. Universidad de Medellín

Se evaluaron las asignaturas de los pregrados con áreas de estudio relacionadas a los del departamento de la Organización de la Universidad Nacional de Colombia, donde se ofrece la asignatura Ética y Voluntariado universitario y Social como componente de libre elección para todas las carreras de pregrado, entre ellas ingeniería administrativa e ingeniería industrial; su intensidad horaria es de cuatro horas presenciales a la semana, donde la metodología y temática se basa en una aproximación conceptual al compromiso ético de la Universidad y el sentido y significado de los programas de voluntariado.

Luego se contextualiza las dinámicas de voluntariados universitarios y se establecen dinámicas de trabajo práctica donde se pone a prueba los valores propuestos en el compromiso ético de la Universidad nacional de Colombia. (Universidad Nacional, 2017).

Los resultados de las otras universidades se describen a continuación:

En la Universidad de Antioquia se evalúan los planes de estudio de ingeniería industrial y administración de empresas, en los cuales ninguno cuenta con materias asociadas a ética, moralidad, humanismo, entre otras.(Universidad de Antioquia, 2017) 


\section{Contexto valencia, $s_{\text {, Roas }, M, \text { vera, }}$}

Para la Universidad Eafit se analizan las carreras Ingeniería de producción, Negocios internacionales y Administración de negocios por ser áreas afines a las trabajadas en la Facultad de Minas.

En los resultados, ingeniería de producción no cuenta con materias éticas en la malla curricular; sin embargo, para negocios internacionales y administración de negocios ofrecen en modalidad de asignatura complementaria las materias (EAFIT, 2017):

- Ética

- Ética y Responsabilidad Social

- Ética y responsabilidad social del contador

Además, EAFIT cuenta con un Núcleo de formación institucional, en el cual se trabajan áreas de ciencias y humanidades, desarrollando las habilidades comunicativas, conociendo el contexto colombiano, la constitución, ciudadanía y ampliando conocimientos de emprendimiento. Éste es un ciclo obligatorio para todos los pregrados de la universidad, las materias son dictadas en forma de cátedras presenciales con una intensidad horaria de tres horas semanales (EAFIT, 2017).

En el caso de la Universidad pontificia Bolivariana - UPB - se analizan las carreras ingeniería administrativa e ingeniería industrial, las cuales cuentan con un área de formación llamada Ciclo básico de formación humanista, este consta de las siguientes materias (Universidad Pontificia Bolivariana, 2017).

Para la Universidad de Medellín se investigan cuatro programas de pregrado, lo cuales están asociados con la escuela de la organización en la facultad de Minas, Medellín. Estos son:

- Ingeniería Industrial

- Ingeniería Financiera

- Negocios Internacionales

- Administración de empresas 
En ingeniería financiera cuentan con la asignatura Ética y en administración de empresas la asignatura Ética y responsabilidad social empresarial, mientras que en ingeniería industrial y negocios internacionales no poseen ninguna materia obligatoria referente a las ciencias éticas (Universidad de Medellín, 2016).

Los objetivos principales de estas materias son, dar a conocer los conceptos generales de la ética, cómo se relacionan con las organizaciones y los negocios, y cómo se da la gestión empresarial de forma ética. Las asignaturas se cursan de manera presencial con una intensidad de cuatro horas por semana. (Universidad de Medellín, 2016).

Como conclusión de este estudio, se obtiene:

- El $80 \%$ de las universidades de Medellín ofrecen alguna materia relacionada con Ciencias Éticas para sus estudiantes de pregrado.

- Las asignaturas analizadas son primordialmente presenciales.

- Los temas están enfocados a los aspectos teóricos de la ética, comprensión de conceptos y contextualización social.

- La intensidad horaria de las asignaturas varía entre tres y cuatro horas de clase por semana.

- El $40 \%$ de las universidades ofrecen materias de ciencias éticas como un componente obligatorio del plan de estudios.

\subsection{Modelo de Asignatura Ciencias Éticas en Ingeniería}

Teniendo en cuenta los resultados de las investigaciones anteriores se procede con la formulación de la asignatura de pregrado sobre ciencias éticas para el Departamento de ingeniería la Organización de la Universidad Nacional de Colombia, sede Medellín.

Para esto, se tiene en cuenta el método de adquisición de la información por los estudiantes como un factor relevante del aprendizaje, así: 
Valencia, S., Rojas, M. y Vera, E.

Toda lo retenido por el cerebro depende directamente de la forma en la que se adquiere, ver Tabla 1.

Tabla1. Porcentaje de información retenida según la actividad.

\begin{tabular}{lc}
\hline \multicolumn{1}{c}{ Actividad } & $\begin{array}{c}\text { Porcentaje de } \\
\text { información retenida }\end{array}$ \\
\hline Leer & $10 \%$ \\
Escuchar & $20 \%$ \\
Visualizar & $30 \%$ \\
Escuchar + Visualizar & $50 \%$ \\
Discutir & $70 \%$ \\
Hacer & $90 \%$ \\
\hline
\end{tabular}

Robson, 2010

Es por esto, que el modelo de asignatura para ciencias éticas se enfoca en discutir y desarrollar casos prácticos, donde los estudiantes afronten situaciones que podrían evidenciar como un juicio moral en su vida laboral futura.

Otra característica principal en la asignatura, será desarrollar apropiadamente los aspectos educativos mencionados en la Figura 2, principalmente las habilidades intelectuales, las estrategias cognoscitivas, información verbal y las actitudes.

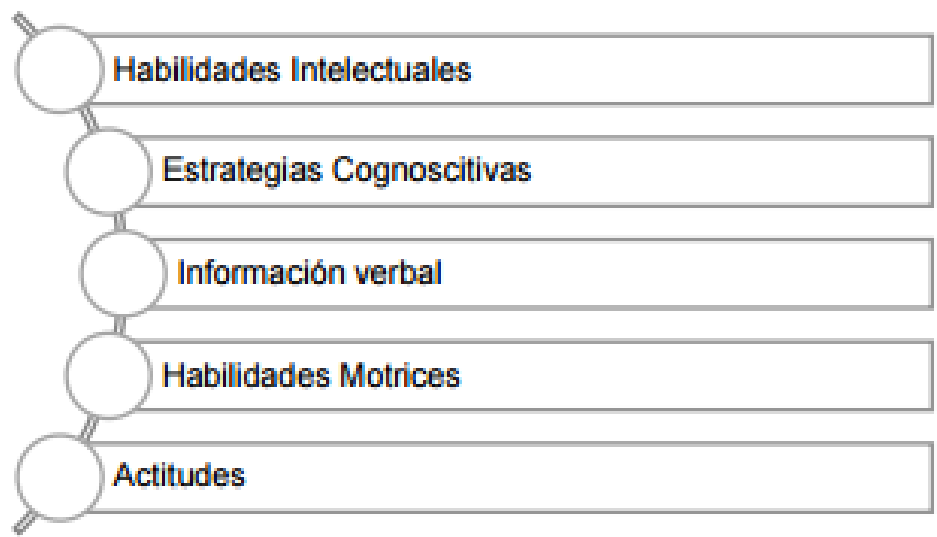

Figura 2. Aspectos educativos.

Rodas, 2004 
Habilidades intelectuales: son las destrezas desarrolladas por el cerebro para resolver un problema o un caso. Permiten responder a los estímulos del entorno. El ciclo de solución de un problema se presenta a continuación:

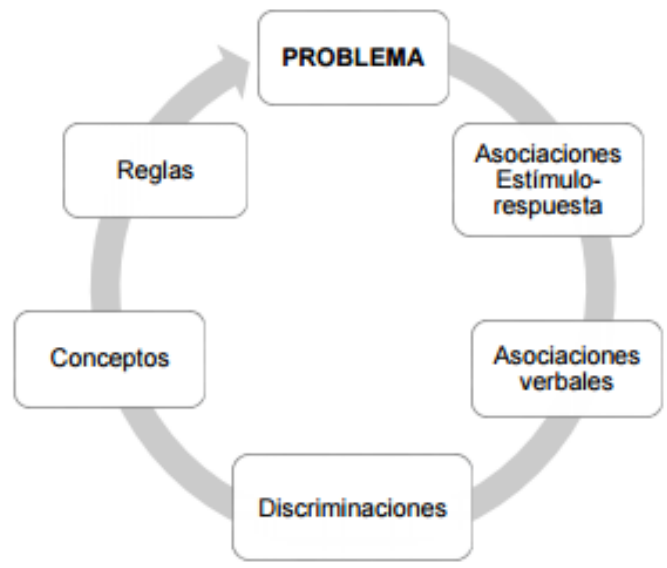

Figura 3. Solución de un problema.

Rodas, 2004

Las asociaciones de los estímulos respuesta y verbales son niveles inferiores de aprendizaje y se desarrollan en la infancia, luego se aprende a discriminar el problema, identificar la clase a la que pertenece y si tiene rasgos en común con otros problemas a los que ya se ha enfrentado. La universidad participa directamente en este aspecto.

Además de las discriminaciones, la universidad otorga conceptos concretos para los problemas y un sistema de solución (reglas), las cuales tienen dos objetivos (Rodas, 2004):

- Recordar al estudiante conceptos fundamentales.

- Disponer de estos conceptos de manera adecuada, -primero definir un sistema, analizar el estado, analizar el ciclo-.

Esto crea un método de solución mental de acuerdo al problema que se esté enfrentando, administración, costos, contabilidad, logística, matemática, programación, entre otros. 


\section{Contexto Valencia, S, Rojas, M. . y Vera, E}

Estrategias cognoscitivas: son capacidades intelectuales específicas que se desarrollan de acuerdo al área de desempeño del estudiante, donde el proceso es: 1-atender, 2-aprender, 3- recordar y 4-pensar (Rodas, 2004).

El aprendizaje de estas estrategias es individual y se da por descubrimiento de aspectos, por lo tanto, es una cualidad en la cual los docentes no deben interferir.

Sin embargo, al educar por medio de experiencias se incita al estudiante a redescubrir los conocimientos y generar estrategias cognoscitivas en el cerebro, dejando de un lado el aprendizaje memorístico (Rodas, 2004).

Información verbal: es la información almacenada en el cerebro con contenido verbal, palabras, nombres, lugares, hechos históricos, entre otras (Rodas, 2004).

Habilidades motrices: habilidades desarrolladas en los primeros años de vida o con práctica en la vida adulta; en estas la familia tiene un alto impacto, son tales como: caminar, escribir, dibujar, usar un abrelatas, manejar, entre otras. Algunas se ven mejoradas con la práctica en la universidad, tal como el dibujo para un arquitecto o la escritura para un periodista (Rodas, 2004).

Actitudes: son estados internos de la persona que influyen directamente en la conducta. Son el resultado del aprendizaje de las personas de la interacción social, generan reacciones positivas o negativas ante las situaciones (Rodas, 2004).

Tomando estos conceptos, la asignatura de ciencias éticas que se desarrolla se enfoca en:

-Incentivar la discusión y el hacer individual en los estudiantes.

-Desarrollar habilidades intelectuales y propiciar estrategias cognoscitivas por medio de experiencias.

- Evitar el aprendizaje memorístico.

- Aumentar el compromiso social y los valores humanos en la formación profesional. $\bullet$ Motivar el enfoque de aprendizaje profundo y la conciencia del 
Revisión de Formación Ética en la Universidad...

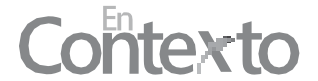

acto de aprendizaje por medio de modificaciones en el contexto del aprendizaje (metodología y material de aprendizaje).

Para esto se utiliza un Método de enseñanza cooperativa donde se forman grupos de trabajo con compañeros desconocidos -aleatorios- de un máximo de 5 personas por grupo. A cada equipo se le entrega un problema con una situación de discusión ética real que esté relacionada con su área de aprendizaje, para este caso particular - Departamento de Ingeniería de la Organización de la Facultad de Minas- serían problemas de negocios, toma de decisiones con empleados, decisiones de automatización y despido de personal, entre otros (Rodas, 2004)(Mátraházi, 2003).

Los problemas no tienen una solución establecida, pero se pretende que de acuerdo a los conceptos básicos de moralidad y ética que se presentan al inicio del curso, cada grupo tome la decisión para resolver la discusión planteada y luego analicen todos los puntos de vista y soluciones posibles.

Al finalizar el análisis, un responsable de cada equipo expone al resto del salón la solución que han planteado, cada una de estas se escriben en el tablero y se realiza una discusión general con todos los estudiantes en la cual se toma la decisión definitiva.

Los equipos de trabajo serán diferentes para cada sesión de clase, así los estudiantes al finalizar el curso habrán trabajado con todos sus compañeros y escuchado sus diferentes opiniones.

El cronograma de la clase será, ver Tabla 2: 
Tabla 2. Cronograma de la asignatura.

\begin{tabular}{|c|c|c|}
\hline Act & Descripción & Duración \\
\hline 1 & $\begin{array}{l}\text { Saludo, comentario de } \\
\text { noticias de actualidad e } \\
\text { información general sobre los } \\
\text { sucesos de la semana. }\end{array}$ & $15 \mathrm{~min}$ \\
\hline 2 & Formación de los equipos & $10 \mathrm{~min}$ \\
\hline 3 & Análisis del caso por equipo & $30 \mathrm{~min}$ \\
\hline 4 & $\begin{array}{l}\text { Exposición de solución del } \\
\text { caso por equipos. }\end{array}$ & $30 \mathrm{~min}$ \\
\hline 5 & $\begin{array}{l}\text { Análisis grupal de las } \\
\text { soluciones planteadas. }\end{array}$ & $15 \mathrm{~min}$ \\
\hline 6 & Conclusión final de la sesión. & $5 \mathrm{~min}$ \\
\hline 7 & $\begin{array}{l}\text { Evaluación de compañeros } \\
\text { del grupo. }\end{array}$ & $5 \mathrm{~min}$ \\
\hline & Tiempo total & $110 \mathrm{~min}$ \\
\hline
\end{tabular}

Elaboración propia.

En el curso será obligatoria la asistencia, por tanto, esto tendrá un porcentaje en la evaluación final del estudiante; también se calificará la participación en la clase y disposición de participación. Al ser un método de enseñanza cooperativa, cada estudiante evaluará a sus compañeros de equipo de la sesión. Esta evaluación no calificará las opiniones que se dieron en la discusión, será enfocada a la participación y motivación del compañero para realizar la actividad adecuadamente.

El profesor en la asignatura sólo será un guía del proceso, no impondrá su opinión ante las situaciones, sólo participará con experiencia. Cada clase la dirige un profesor diferente de la facultad, así los estudiantes podrán aprender de múltiples maestros, diferentes puntos de vista y modelos para solucionar un problema.

La calificación del docente no será relevante a la hora de evaluar al estudiante, ver Tabla 3. 
Tabla 3. Evaluación de la asignatura Ciencias Éticas.

\begin{tabular}{lc}
\hline \multicolumn{1}{c}{ Descripción } & Porcentaje \\
\hline Asistencia & $40 \%$ \\
$\begin{array}{l}\text { Promedio de evaluación por parte } \\
\text { de compañeros de trabajo. }\end{array}$ & $60 \%$ \\
\hline
\end{tabular}

Elaboración propia.

\section{Información General de la Asignatura}

Nivel: Pregrado.

Nombre: Ciencias éticas.

Unidad Académica básica: Escuela de la organización, Facultad de Minas. Horas presenciales: Dos (2) horas por semana.

Número de Créditos: Dos (2)

Semestre: Octavo en adelante

Modalidad: Obligatoria para ingeniería administrativa e ingeniería industrial. Descripción: La asignatura proporcionará a los estudiantes herramientas básicas para forjar la toma de decisiones en un nivel laboral, por medio de análisis de casos y opiniones; además los enfrentará a situaciones de posible ocurrencia en el ámbito profesional, para los cuales tendrán unas bases éticas objetivas, defendiendo los valores humanos sobre cualquier escenario.

Contenido (Díaz Rodríguez, 2006)(National Society of Professional Engineers, 2016)

1. Introducción al curso

1.1. Ética

1.2. Moral

1.3. Ética como ciencia

1.4. Ética profesional

2. Código de ética para ingenieros

2.1. Normas fundamentales

2.2. Reglas para la práctica de la ingeniería 2.3. Obligaciones profesionales

3. Casos de discusión 


\section{Contexto valencia,, , fojas, M. vera, E.}

\section{Conclusiones}

La sociedad colombiana se encuentra en un proceso de transformación, que exige a los participantes trabajar bajo lineamientos éticos para ser agentes activos del cambio. La Facultad de Minas de la Universidad Nacional de Colombia, Sede Medellín, desde el año 1912 trabaja bajo el lema "Trabajo y Rectitud" propuesto por el ingeniero Juan de la Cruz Posada. Analizándolo, se entiende por trabajo la disciplina, rigor y exactitud que debe tener el ingeniero, y en general todos los profesionales, en las labores que desempeñe en su diario ejercicio de la profesión. Rectitud hace referencia a la base fundamental de la formación de los profesionales en valores como honestidad y responsabilidad que comprenden su compromiso y responsabilidad con la sociedad.

Se evidencian falencias en las Universidades de Medellín que fueron objeto de estudio, en cuanto a cursos con contenido en ética, lo cual afecta la efectividad de aprendizaje del estudiante y no permite que durante la formación profesional se presente un acercamiento con la problemática y dilemas éticos presentes en el ejercicio de la profesión.

Los métodos actuales de enseñanza deben ser revisados debido a que la tecnología ha evolucionado para mejorar la didáctica en el proceso enseñanzaaprendizaje.

La enseñanza cooperativa es apropiada cuando el aprendizaje debe modificar aspectos cognoscitivos del estudiante, como capacidad de análisis, interacción social, toma de decisiones, criterio personal, pensamiento crítico y formación del carácter.

Es importante propiciar espacios que incentiven la discusión y que propongan al estudiante como centro del aprendizaje, permitiendo desarrollar capacidades sociales y compromisos éticos, complementos necesarios en un perfil profesional.

La propuesta se fundamenta por la ausencia de formación en el estudiante y el énfasis técnico de las facultades en el conocimiento disciplinar. 


\section{Referencias}

Buendía Eisman, L., \& Olmedo Moreno, E. M. (2002). El género: ¿constructo mediador en los enfoques de aprendizaje universitario? Revista de Investigación Educativa, 20(2), 511-524. Retrieved from http://dialnet.unirioja.es/servlet/oaiart?codigo $=305224$

Díaz Rodríguez, M. . (2006). Programa de Asignatura Ética Profesional.

EAFIT, U. (2017). Oferta de Pregrados en EAFIT. Retrieved May 8, 2017, from http://www.eafit.edu.co/programasacademicos/Paginas/pregrados.aspx.

Entwistle, N. (1991). La Comprensión del aprendizaje en el aula. PAIDOS IBERICA.

Marton, F., \& Lennart, S. (1979). Conceptions of research in student learning. Higher Education, 8(4), 471-486.

Mátraházi, N. (2003). El método cooperativo aplicado en la clase de ELE en la Enseñanza Secundaria Obligatoria y en la enseñanza de adultos en Hungría. Instituto Bilingüe Húngaro-Español Károlyi Mihály de Budapest, 271-278.

Molina Álvarez, A. T. (1999). Problemática actual en la enseñanza de la ingeniería: una alternativa para su solución. Ingenierías, II(3), 10-15. Retrieved from http://www.ingenierias.uanl.mx/7/pdf/7_Ana_T_Molina_Problematica_actual. pdf

National Society of Professional Engineers, N. (2016). NSPE Code of Ethics for Engineers. Retrieved May 8, 2017, from www.nspe.org/resources/ethics/codeethics

QS Top University, "QS University Rankings: Latin America." (2015). Retrieved May 8, 2017, from /www.topuniversities.com/latinamerican-rankings/digitalsupplement-2015.

Robson, G. D. (2010). Continuous Process Improvement. Toronto: The free press. 


\section{Contexto valencia, S., Rojas, M. y vera, E.}

Rodas, J. M. (2004). INVESTIGACIÓN SOBRE MÉTODOS DE ENSEÑANZA-. Facultad de Ingeniería - Universidad Rafael Landivar, (04), 1-16.

Serna M, E. (2015). Por qué falla el sistema de educación. Retrieved from http://fundacioniai.org/Libro4.pdf

Universidad de Antioquia, M. (2017). Oferta de Programas de Pregrado. Retrieved May 8 , 2017 , from http://portal.udea.edu.co/wps/portal/udea/web/inicio/estudiarudea/pregrado/oferta/

Universidad de Medellín, U. (2016). Oferta Académica. Retrieved May 8, 2017, from http://www.udem.edu.co/index.php/ofertaacademica-completa-de-la-udem.

Universidad Nacional, M. (2017). Buscador de asignaturas y cursos. Retrieved May 8,2017 , from

http://sia2.medellin.unal.edu.co:9401/buscador/service/action.pub.

Universidad Pontificia Bolivariana, U. (2017). Progradas de Pregrado UPB. Retrieved May 8, 2017, from http://www.upb.edu.co/portal/page?_pageid=1054,28639268\&_dad=portal

Zerpa, C. E. (2012). TICA, CAPITAL SOCIAL Y DESARROLLO EN LA FORMACIÓN PROFESIONAL DE LA CARRERA DE INGENIERÍA: UNA PROPUESTA PEDAGÓGICA. Revista de La Facultad de Ingeniería Universidad Central de Venezuela, 27(3). 